Robotic Telescopes, Student Research and Education (RTSRE) Proceedings

Conference Proceedings, San Diego, California, USA, Jun 18-21, 2017

Fitzgerald, M., James, C.R., Buxner, S., White, S., Eds. Vol. 1, No. 1, (2018) ISBN 978-0-6483996-0-5 / doi : 10.32374/rtsre.2017.031 / CC BY-NC-ND license

Peer Reviewed Article. rtsre.net/ojs

\title{
Processing Images from the Zwicky Transient Facility
}

Russ R. Laher ${ }^{1 *}$, Frank J. Masci ${ }^{1}$, Steve Groom ${ }^{1}$, Benjamin Rusholme ${ }^{1}$, David L. Shupe ${ }^{1}$, Ed Jackson $^{1}$, Jason Surace ${ }^{1}$, Dave Flynn ${ }^{1}$, Walter Landry ${ }^{1}$, Scott Terek ${ }^{1}$, George Helou ${ }^{1}$, Ron Beck $^{1}$, Eugean Hacopians ${ }^{2}$, Umaa Rebbapragada ${ }^{3}$, Brian Bue ${ }^{3}$, Roger M. Smith ${ }^{4}$, Richard G. Dekany ${ }^{4}$, Adam A. Miller ${ }^{5}$, S. B. Cenko ${ }^{6}$, Eric Bellm ${ }^{7}$, Maria Patterson ${ }^{7}$, Thomas Kupfer ${ }^{8}$, Lin Yan $^{8}$, Tom Barlow ${ }^{8}$, Matthew Graham ${ }^{8}$, Mansi M. Kasliwal ${ }^{8}$, Thomas A. Prince ${ }^{8}$ and Shrinivas R. Kulkarni ${ }^{8}$

\begin{abstract}
The Zwicky Transient Facility is a new robotic-observing program, in which a newly engineered 600-MP digital camera with a pioneeringly large field of view, 47 square degrees, will be installed into the 48-inch Samuel Oschin Telescope at the Palomar Observatory. The camera will generate $\sim 1$ petabyte of raw image data over three years of operations. In parallel related work, new hardware and software systems are being developed to process these data in real time and build a long-term archive for the processed products. The first public release of archived products is planned for early 2019, which will include processed images and astronomical-source catalogs of the northern sky in the $g$ and $r$ bands. Source catalogs based on two different methods will be generated for the archive: aperture photometry and point-spread-function fitting.
\end{abstract}

\section{Keywords}

asteroids; stars: variables, binaries, supernovae, cataclysmic variables; galactic: active nuclei; techniques: image processing, photometric; methods: observational, data analysis

${ }^{1}$ IPAC, Mail Code 100-22, Caltech, 1200 E. California Blvd., Pasadena, CA 91125, U.S.A.

${ }^{2}$ Anre Technologies Inc., 3115 Foothill Blvd., Suite M202, La Crescenta, CA 91214, U.S.A.

${ }^{3}$ Jet Propulsion Laboratory, California Institute of Technology, Pasadena, CA 91109, U.S.A.

${ }^{4}$ Caltech Optical Observatories, California Institute of Technology, Pasadena, CA 91125, U.S.A.

${ }^{5}$ Center for Interdisciplinary Exploration and Research in Astrophysics, Northwestern University, Evanston, IL 60208, U.S.A.

${ }^{6}$ Astrophysics Science Division, NASA Goddard Space Flight Center, Code 661, Greenbelt, MD 20771, U.S.A.

${ }^{7}$ Department of Astronomy, University of Washington, Seattle, WA 98195, U.S.A.

${ }^{8}$ Division of Physics, Mathematics, and Astronomy, California Institute of Technology, Pasadena, CA 91125, U.S.A. *Corresponding author: laher@ipac.caltech.edu

\section{Introduction}

The Zwicky Transient Facility $\left(\mathrm{ZTF}^{1}\right)$ is a new program for ground-based, optical, time-domain astronomy (Bellm et al., 2015), which goes well

\footnotetext{
${ }^{1}$ http://www.ptf.caltech.edu/ztf
}

beyond its immensely successful predecessor programs, the Palomar Transient Factory $\left(\mathrm{PTF}^{2}\right)$ and eponymous intermediate program (iPTF). A digital camera specially developed for the ZTF, with a faster readout time and a much larger field

${ }^{2}$ http://www.ptf.caltech.edu 
of view, along with other enhancements relative to the PTF camera, such as a fast exposure shutter, is nearing the completion of its development phase, and installation into the 48-inch Samuel Oschin Telescope at the Palomar Observatory is planned for late summer of 2017. Substantial telescope and dome upgrades are part of this effort, including faster drives and improved optics. In addition, the parallel development of hardware and software systems that can handle real-time post-processing of the massive amount of image data from the camera in normal operations, as well as the development of a long-term archive for the processed products, is underway.

The data processing will be performed at IPAC and the ZTF archive will be hosted by the NASA/IPAC Infrared Science Archive (IRSA ${ }^{3}$ ). The data-processing system and archive will rely heavily on relational databases to consolidate and warehouse information that can be later queried in a historical context for the discovery of astrophysical transient phenomena. The major science goals for the ZTF include discovering young supernovae, searching for electromagnetic counterparts to gravitational wave sources, identifying stellar variables, and detecting near-Earth asteroids.

In the Project Background Section, we give background information on the ZTF project, some camera details, and highlights of the observing strategy. In the Data Processing Section, the focus of this paper, we outline our design of the ZTF data-processing system and discuss our recent performance-test results. We emphasize that the system design is still evolving and the results up to this point in time are preliminary. We briefly describe the ZTF archive in the Product Archive Section. Finally, we provide a summary and outlook in the Conclusion Section.

\section{Project Background}

\section{Programmatics}

The ZTF's predecessor programs, PTF and iPTF, have led to the publication of 185 papers in

\footnotetext{
${ }^{3}$ http://irsa.ipac.caltech.edu
}

peer-reviewed journals (at the time of this writing more PTF/iPTF papers are forthcoming). ${ }^{4}$ As an example, a paper on a regular type II supernova based in part on PTF data was recently published in Nature Physics (Yaron et al., 2017). The iPTF project officially ended in March of 2017. A separate enterprise has been underway since then to reprocess the PTF Galactic-plane data for inclusion in a future public data release.

The ZTF is a consortium of several U.S. and global institutions, known as the "collaboration". 5 Both private funds from the members and public (National Science Foundation) have been received for this project. Work on the ZTF began in earnest in 2014, and the current plan is for the ZTF to collect data for three years after regular operations begin.

Data from the ZTF will fuel research projects for students, postdocs and scientists at institutions in the collaboration and the public at large. There is also a related new initiative for undergraduate research known as the Summer Undergraduate Astronomy Institute (Penprase and Bellm, 2017).

\section{Camera}

Detailed engineering papers on the development of the ZTF camera are given by Smith et al. (2014) and Dekany et al. (2016). A definitive instrument paper is in preparation by Dekany et al., and is due out by end of 2017 or early 2018 (to appear in PASP, special ZTF issue, 2018). First light with the camera permanently mounted to the telescope is scheduled for October 2017.

The ZTF-camera field of view is truly groundbreaking, and it will enable imaging of the entire Palomar sky each night. Figure 1 compares it to the field of view of other large-survey cameras, either currently in operation or under development. Table 1 lists the salient attributes of the camera for the planned observing.

The median image quality delivered by the 48-inch telescope optics on Palomar mountain is

\footnotetext{
${ }^{4}$ The published papers are tracked at http://www.ptf.caltech.edu/iptf

${ }^{5}$ http://www.ptf.caltech.edu/ztf
} 


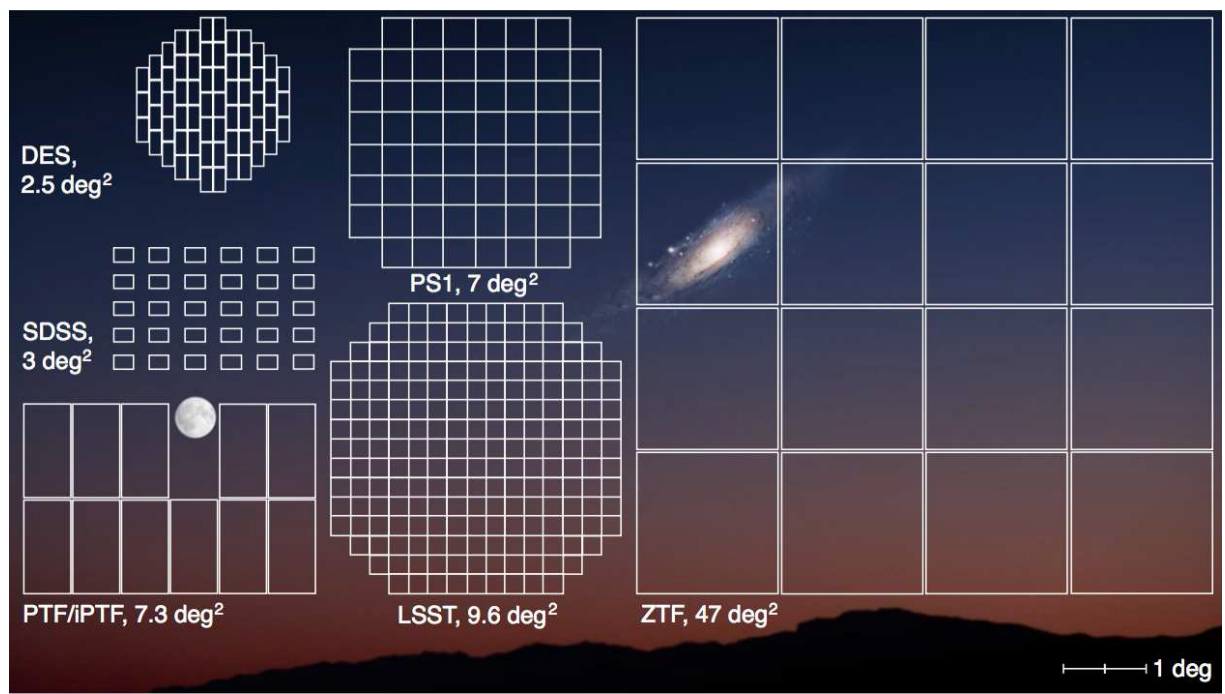

Figure 1. Field of view of the ZTF camera compared to that of other large-survey cameras. The Moon and the Andromeda Galaxy (Messier 31) are shown to scale. (With the permission of Joel Johansson.)

$\approx 2$ arc seconds. This motivated the design choice of 1 arc second Nyquist sampling for the camera.

Table 1. ZTF camera and nominal observing attributes

\begin{tabular}{ll}
\hline \hline Attribute & Value \\
\hline Field of view & 47 square degrees \\
Pixel scale & 1 arc second per pixel \\
Pixel size & $15 \mu \mathrm{m}$ \\
CCD readout channels & 4 \\
Exposure time & 30 seconds \\
Readout time & $\approx 10$ seconds \\
Slew \& settle time & +5 seconds \\
Optical filters & ZTF $g, r$ and $i$ \\
Limiting magnitude & $20.4(r$-band, $5 \sigma)$ \\
Mosaic of CCDs & $4 \times 4$ layout $(16 \mathrm{CCDs})$ \\
Pixels per CCD & $\sim 6 \mathrm{~K} \times 6 \mathrm{~K}$ \\
Total number of pixels & $\approx 600$ megapixels \\
\hline
\end{tabular}

\section{Observing Strategy}

The acquisition of science exposures will occur throughout each observing night, weather permitting. We are budgeting for 260 good-weather nights in our estimates. Depending on the time of year, we expect to acquire 600-800 science exposures per night, assuming one exposure taken every 45 seconds, which allows for 30 -second camera exposures and the fixed readout time and concurrent slew \& settle times of the camera and telescope (see Table 1). Scanning at least 3760 square degrees of the sky per hour will be possible.

Calibration exposures (biases, dome flats, darks, etc.) will be acquired during the day. Fresh calibration products will be made before the nightly processing of science exposures begins.

During the first year and a half of operations, ZTF will conduct two general-purpose public surveys: a three-night cadence survey of the visible Northern Sky, and a nightly sweep of the Galactic Plane. For both programs, fields will be visited twice each night they are observed, with approximately one hour separation between a $g$-band exposure and an $r$-band exposure.

\section{Data Processing}

Laher et al. (2014) describe the image-processing system for the ZTF's predecessor programs, PTF and iPTF. This experience is leveraged in our new design for the ZTF. Below we give an overview of the ZTF Data System. A more detailed description will appear in a future publication (to appear in PASP, special ZTF issue, 2018).

The science exposures will be processed in real time, throughout the observing night. This requires 
a data-processing system that can keep up with the incoming data.

Figure 2 depicts the ZTF processing system that is currently under development at IPAC. It features 64 pipeline machines, four file servers, two database machines (primary and secondary/backup), a private web server, a Kafka ${ }^{6}$ cluster, and an IRSA public web interface. The local network is proficient at data rates of up to 10 gigabits per second.

The pipeline machines are for running ZTF real-time pipelines in parallel. This part of the system is inherently scalable, and more machines can be added as needed. The CPU of each pipeline machine has 16 cores ( 2 threads per core), and 16 pipeline instances per machine are typically run. The open-source, workload-manager software, SLURM $^{7}$, is used to farm out pipeline instances to pipeline machines in the proper order and number. The real-time pipeline generates many interim files at its various steps, and these files typically become inputs to a subsequent step. The interim files are written to the local disks of pipeline machines for speed and avoidance of unnecessary congestion on the local network.

The four file servers allow parallel file transfer across the local network. Files copied from pipeline-machine local disks to and from the sandbox file system are expected to generate the most traffic. Products ultimately copied to the archive file system will add marginally to the load. This part of the system is also scalable and more machines could potentially be added to service additional load.

Our data-processing system features a PostgreSQL ${ }^{8}$ relational database running on the primary database machine, equipped with 384 gigabytes of memory. Since this element is not scalable, careful, disciplined database design, and both hardware and software tuning have proven to be absolutely essential. Database tables and

\footnotetext{
${ }^{6}$ https://kafka.apache.org/documentation

${ }^{7}$ https://slurm.schedmd.com

${ }^{8}$ https://www.postgresql.org
}

indexes are strategically micromanaged so that heavily accessed data are stored on solid-state devices (SSDs) as opposed to spinning disks. The large database-machine memory and judicious database tuning ensure a very high cache-hit rate. Daily database vacuuming is required for regular maintenance. The database is replicated onto a second database machine, so that design-team members can query it without affecting the primary database. Candidate transients are stored in database tables partitioned by observing date and readout channel. A set-up process that creates database views over the correct candidate partitions is executed daily.

The web server will allow ZTF personnel to perform quality analysis on the raw data and processed products. It will also serve as a staging ground for initial access to archive products as well as disseminate products for solar system science.

The Kafka cluster is a set of machines at IPAC and a set of machines at the University of Washington (UW) that facilitates the distribution of transient-event alerts to the ZTF collaboration and eventually the community. Once the event data has been sent to Kafka, which is enabled by pipeline packaging the data in Avro format, ${ }^{9}$ the Kafka software handles mirroring between IPAC and UW over the Internet.

Figure 3 gives a flowchart of the ZTF real-time pipeline. The CCD-image files received from Palomar consist of one multi-extension FITS file for each CCD and exposure. These are split into readout-channel quadrants for smaller images to speed up the subsequent parallel processing, in which the quadrant images are processed independently. Bias and flat-field corrections are applied to the applicable raw images. The processing involves instrumental calibration, which consists of astrometric and photometric calibration, followed by image differencing with a reference image for finding transients. Scamp ${ }^{10}$ is used in conjunction with the GAIA catalog (Brown et al.,

\footnotetext{
${ }^{9}$ E.g., https://avro.apache.org/docs/1.8.2/

${ }^{10} \mathrm{https}$ ///www.astromatic.net/software/scamp
} 


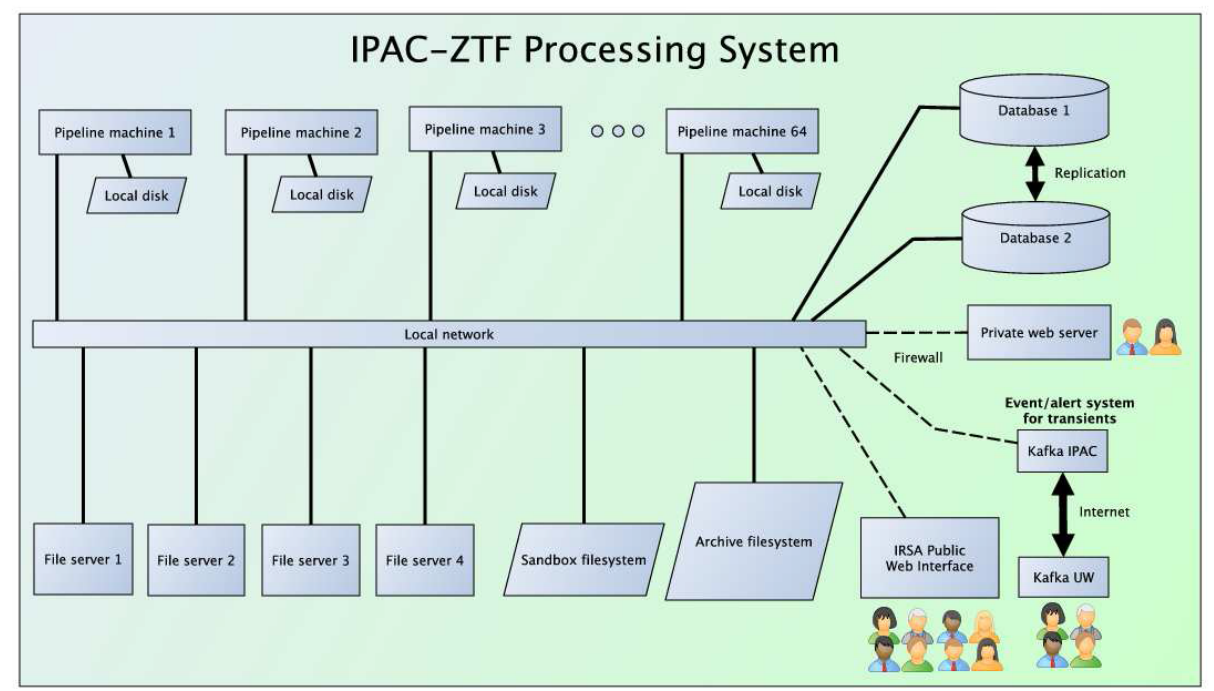

Figure 2. ZTF data-processing system.

2016) to perform the astrometry and compute a World Coordinate System for each science image. Absolute photometry is done using the Pan-STARRS DR1 catalog as the standard (Flewelling, 2017). Reference images, which are needed for image differencing, are made within the first few months of operation. The image differencing includes a combination of the steps described in Masci et al. (2016), as well as an implementation of the ZOGY algorithm (Zackay et al., 2016). Candidate transient events are detected in thresholded difference images, and then vetted with machine learning provided by JPL, for example, using the real/bogus framework of Bloom et al. (2012). Asteroid streaks are found in the difference images using the findstreaks module (Masci et al., 2016), and subsequently checked for reliability using a new deterministic algorithm that accounts for the point-spread function of the streak.

Figure 4 gives preliminary performance test results for the ZTF real-time pipelines processing a night's worth of simulated data. The density plot includes 28,505 independent pipeline instances running on 32 pipeline machines, and shows that a night spanning $\sim 7.5$ hours can be processed in $\sim 6.2$ hours, which is better than real time. The median run time for a pipeline instance in this test is 275.5 seconds. The primary factors that affect the pipeline run times include: 1) The number of astronomical sources extracted from the images (e.g., Galactic-plane observations are much more stressing); and 2) The fraction of images for the night that actually have reference images available (this was $\sim 75 \%$ for our simulated data). The simulated data for the test include random transients at a rate of 10 per CCD. Future tests will involve simulated transient rates that are up to $8 \times$ higher.

\section{Product Archive}

As illustrated in Figure 2, IRSA will set up infrastructure for a long-term archive and a web interface for the distribution of ZTF-survey products to the collaboration and public. The web interface will have similar functionality as those available to IRSA archives for other projects. The entire archive is expected to grow to $\sim 3$ petabytes by the end of ZTF's three-year program. Table 2 lists the product types that will be archived, which will include both images and astronomical-source catalogs. Two different methods will be used to generate source catalogs for the archive, namely, aperture photometry and point-spread-function (PSF) fitting.

The first public release of archived products is planned for early 2019. This release will include only products from the ZTF public surveys. 


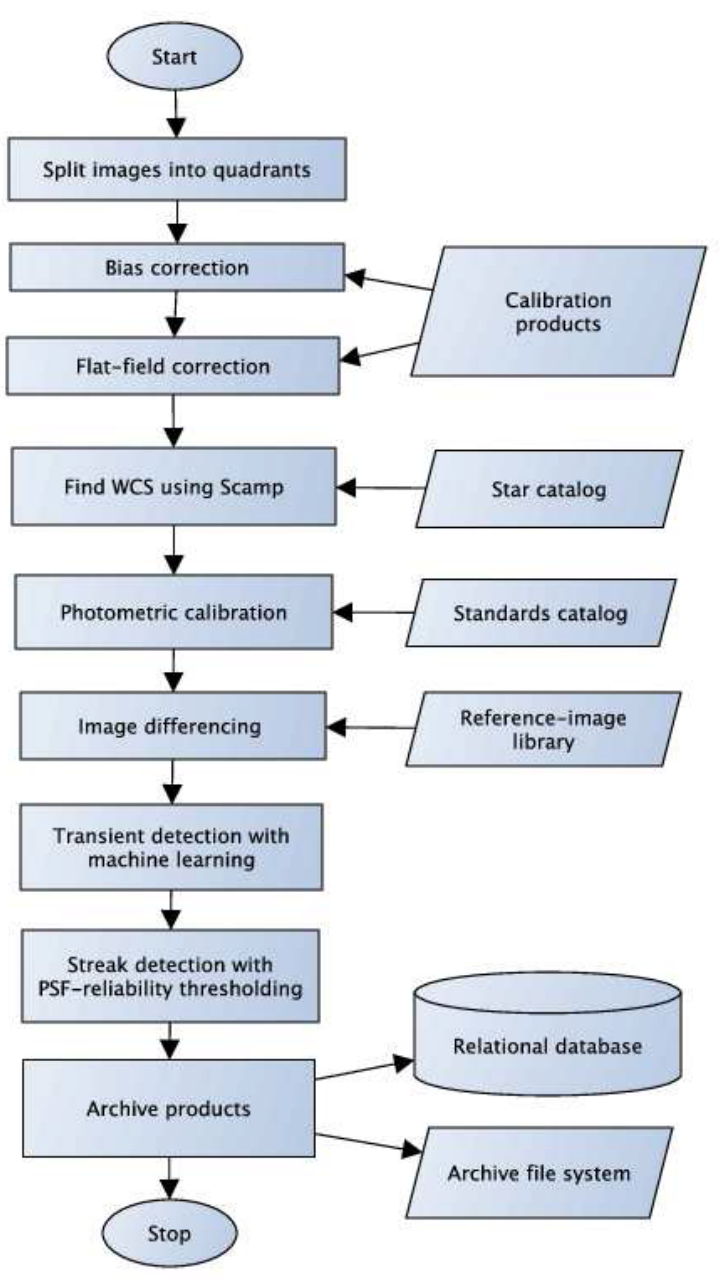

Figure 3. ZTF data-processing system.

Products from collaboration observing programs will be included in later releases.

\section{Conclusion}

This paper gives an overview of the ZTF project, camera, observing strategy, data-processing system, preliminary benchmark results, and product archive, with an emphasis on describing the data-processing system and archive. In the coming months, the data-processing system will be exercised on real data from the observatory-mounted camera. Building upon our past successes gives us confidence in a favorable outcome for this endeavor. Exciting new discoveries await astronomers analyzing ZTF products!
Table 2. ZTF archive products

Product
Raw images
Processed images
Image masks
Difference images
SExtractor catalogs
PSF-fit catalogs (DAOPHOT)
Reference images, catalogs, etc.
Calibration products
Light curves

\section{Acknowledgements}

We are grateful to Joel Johansson of the Oskar Klein Center at Stockholm University and the Weizmann Institute of Science for permission to use the original creative work shown in Figure 1.

ZTF is led by the California Institute of Technology, US and includes IPAC, US; the Joint Space-Science Institute (via the University of Maryland, College Park), US; Oskar Klein Centre of the University of Stockholm, Sweden;

University of Washington, US; Weizmann Institute of Science, Israel; DESY and Humboldt University of Berlin, Germany; University of Wisconsin at Milwaukee, US; the University System of Taiwan, Taiwan; and Los Alamos National Laboratory, US; ZTF acknowledges the generous support of the National Science Foundation under AST MSIP Grant No 1440341. The alert distribution service is provided by the DIRAC Institute at the University of Washington.

Part of this research was carried out at the Jet Propulsion Laboratory, California Institute of Technology, under a contract with the National Aeronautics and Space Administration.

\section{References}

Bellm, E. C., Kulkarni, S. R., Collaboration, Z., et al. (2015). The Zwicky Transient Facility. In American Astronomical Society Meeting Abstracts, volume 225.

Bloom, J., Richards, J., Nugent, P., Quimby, R., Kasliwal, M., Starr, D., Poznanski, D., Ofek, E., 


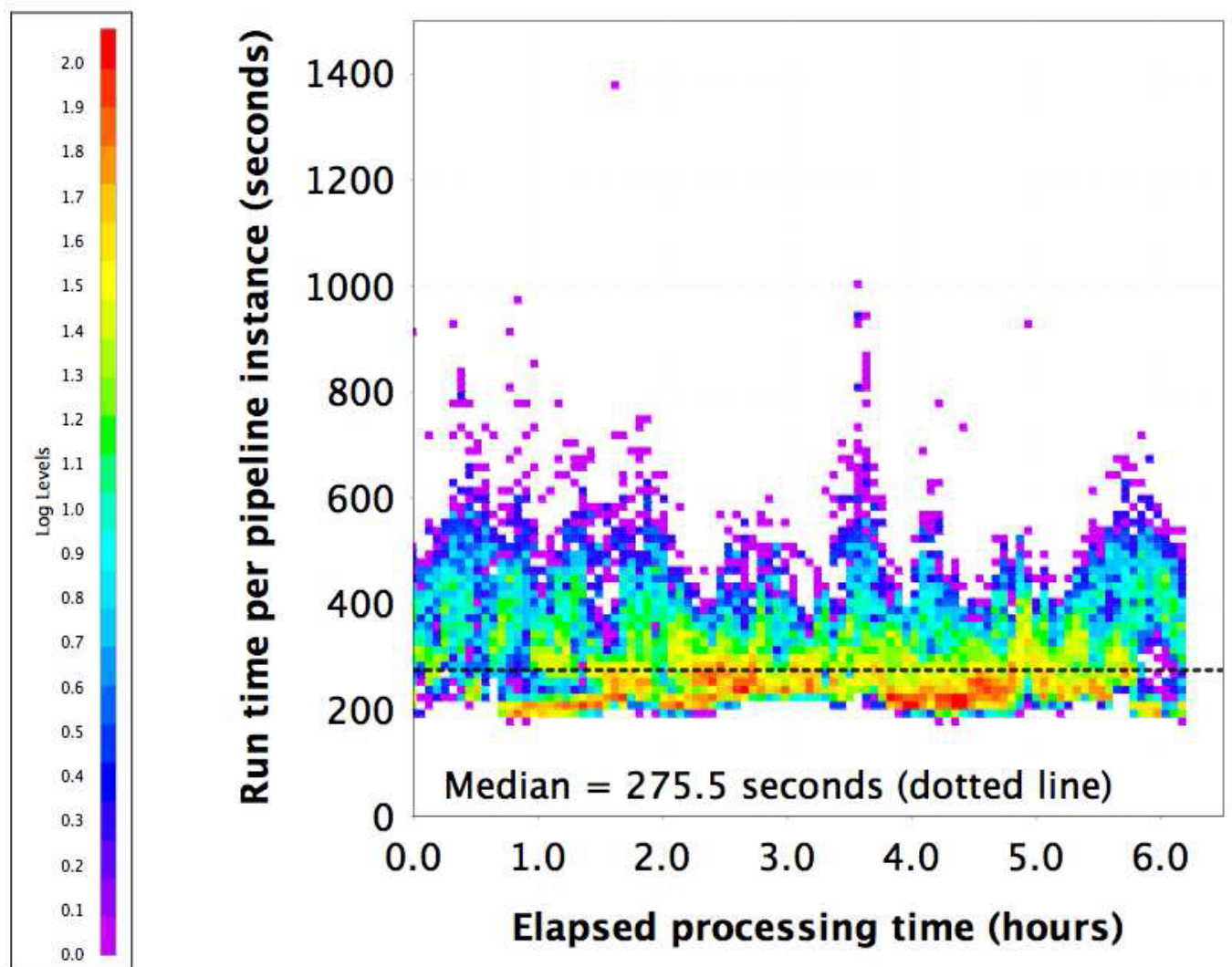

Figure 4. Preliminary ZTF benchmark-test results.

Cenko, S., Butler, N., et al. (2012). Automating discovery and classification of transients and variable stars in the synoptic survey era. Publications of the Astronomical Society of the Pacific, 124(921): 1175.

Brown, A. G., Vallenari, A., Prusti, T., De Bruijne, J., Mignard, F., Drimmel, R., Babusiaux, C., Bailer-Jones, C., Bastian, U., Biermann, M., et al. (2016). Gaia Data Release 1-Summary of the astrometric, photometric, and survey properties. Astronomy \& Astrophysics, 595:A2.

Dekany, R., Smith, R. M., Belicki, J., Delacroix, A., Duggan, G., Feeney, M., Hale, D., Kaye, S., Milburn, J., Murphy, P., et al. (2016). The Zwicky Transient Facility Camera. In Ground-based and Airborne Instrumentation for Astronomy VI, volume 9908, page 99085M. International Society for Optics and Photonics.

Flewelling, H. (2017). Pan-STARRS Data Release
1. In American Astronomical Society Meeting Abstracts, volume 229.

Laher, R. R., Surace, J., Grillmair, C. J., Ofek, E. O., Levitan, D., Sesar, B., van Eyken, J. C., Law, N. M., Helou, G., Hamam, N., et al. (2014). IPAC image processing and data archiving for the Palomar Transient Factory. Publications of the Astronomical Society of the Pacific, 126(941):674.

Masci, F. J., Laher, R. R., Rebbapragada, U. D., Doran, G. B., Miller, A. A., Bellm, E., Kasliwal, M., Ofek, E. O., Surace, J., Shupe, D. L., et al. (2016). The IPAC image subtraction and discovery pipeline for the Intermediate Palomar Transient Factory. Publications of the Astronomical Society of the Pacific, 129(971):014002.

Penprase, B. E. and Bellm, E. C. (2017). ZTF Undergraduate Astronomy Institute at Caltech and Pomona College. In American Astronomical Society Meeting Abstracts, volume 229. 
Smith, R. M., Dekany, R. G., Bebek, C., Bellm, E., Bui, K., Cromer, J., Gardner, P., Hoff, M., Kaye, S., Kulkarni, S., et al. (2014). The Zwicky transient facility observing system. In Ground-based and Airborne Instrumentation for Astronomy V, volume 9147, page 914779. International Society for Optics and Photonics.

Yaron, O., Perley, D., Gal-Yam, A., Groh, J., Horesh, A., Ofek, E., Kulkarni, S., Sollerman, J., Fransson, C., Rubin, A., et al. (2017). Confined dense circumstellar material surrounding a regular type II supernova. Nature Physics, 13(5):510.

Zackay, B., Ofek, E. O., and Gal-Yam, A. (2016). Proper Image Subtraction-Optimal Transient Detection, Photometry, and Hypothesis Testing. The Astrophysical Journal, 830. 